(1)

CrossMark

\title{
The Pleural Effusion And Symptom Evaluation (PLEASE) study of breathlessness in patients with a symptomatic pleural effusion
}

\author{
Sanjeevan Muruganandan (1012,3,15, Maree Azzopardi ${ }^{4,15}$, Rajesh Thomas ${ }^{2,3,5}$, \\ Deirdre B. Fitzgerald ${ }^{2,3,5}$, Yi Jin Kuok ${ }^{6}$, Hui Min Cheah ${ }^{2,3}$, Catherine A. Read ${ }^{2,3}$, \\ Charley A. Budgeon 7,8 , Peter R. Eastwood (10,10,11, Susan Jenkins ${ }^{12,13,14}$, \\ Bhajan Singh ${ }^{9,10,11}$, Kevin Murray ${ }^{8}$ and Y.C. Gary Lee Le,3,5 $^{2,5}$
}

@ERSpublications

The majority of patients improve after pleural fluid drainage. Abnormal diaphragmatic function may be an important contributor to breathlessness in patients with pleural effusion. http://bit.ly/2SyF8RW

Cite this article as: Muruganandan S, Azzopardi M, Thomas R, et al. The Pleural Effusion And Symptom Evaluation (PLEASE) study of breathlessness in patients with a symptomatic pleural effusion. Eur Respir J 2020; 55: 1900980 [https://doi.org/10.1183/13993003.00980-2019].

\section{ABSTRACT}

Introduction: Pathophysiology changes associated with pleural effusion, its drainage and factors governing symptom response are poorly understood. Our objective was to determine: 1) the effect of pleural effusion (and its drainage) on cardiorespiratory, functional and diaphragmatic parameters; and 2) the proportion as well as characteristics of patients with breathlessness relief post-drainage.

Methods: Prospectively enrolled patients with symptomatic pleural effusions were assessed at both pretherapeutic drainage and at $24-36 \mathrm{~h}$ post-therapeutic drainage.

Results: 145 participants completed pre-drainage and post-drainage tests; $93 \%$ had effusions $\geqslant 25 \%$ of hemithorax. The median volume drained was $1.68 \mathrm{~L}$. Breathlessness scores improved post-drainage (mean visual analogue scale (VAS) score by $28.0 \pm 24 \mathrm{~mm}$; dyspnoea-12 (D12) score by $10.5 \pm 8.8$; resting Borg score before 6 -min walk test $(6-\mathrm{MWT})$ by $0.6 \pm 1.7$; all $\mathrm{p}<0.0001)$. The 6 -min walk distance $(6-\mathrm{MWD})$ increased by $29.7 \pm 73.5 \mathrm{~m}, \mathrm{p}<0.0001$. Improvements in vital signs and spirometry were modest (forced expiratory volume in $1 \mathrm{~s}\left(\mathrm{FEV}_{1}\right)$ by $0.22 \mathrm{~L}, 95 \% \mathrm{CI} 0.18-0.27$; forced vital capacity (FVC) by $0.30 \mathrm{~L}, 95 \% \mathrm{CI} 0.24-0.37$ ). The ipsilateral hemi-diaphragm was flattened/everted in 50\% of participants pre-drainage and $48 \%$ of participants exhibited paradoxical or no diaphragmatic movement. Post-drainage, hemi-diaphragm shape and movement were normal in $94 \%$ and $73 \%$ of participants, respectively. Drainage provided meaningful breathlessness relief (VAS score improved $\geqslant 14 \mathrm{~mm}$ ) in $73 \%$ of participants irrespective of whether the lung expanded (mean difference $0.14,95 \%$ CI 10.02-0.29; $\mathrm{p}=0.13$ ). Multivariate analyses found that breathlessness relief was associated with significant breathlessness pre-drainage (odds ratio (OR) 5.83 per standard deviation (SD) decrease), baseline abnormal/paralyzed/paradoxical diaphragm movement (OR 4.37), benign aetiology (OR 3.39), higher pleural $\mathrm{pH}$ (OR per SD increase 1.92) and higher serum albumin level (OR per SD increase 1.73). Conclusions: Breathlessness and exercise tolerance improved in most patients with only a small mean improvement in spirometry and no change in oxygenation. Breathlessness improvement was similar in participants with and without trapped lung. Abnormal hemi-diaphragm shape and movement were independently associated with relief of breathlessness post-drainage.

This article has an editorial commentary: https://doi.org/10.1183/13993003.00501-2020

This article has supplementary material available from erj.ersjournals.com

This study is registered at https://www.anzctr.org.au/ with identifier number ACTRN12616000820404.

Received: 15 May 2019 | Accepted after revision: 5 Feb 2020

Copyright @ERS 2020 


\section{Introduction}

Pleural effusion affects an estimated 24 million people annually worldwide [1]. Breathlessness is the most common presenting symptom and is often disabling and requires invasive drainage procedures [2]. The pathophysiologic mechanism(s) of breathlessness associated with pleural effusion are complex, multifactorial and poorly understood. Several small studies [3-5] show that improvements in lung function following drainage of effusions are modest and correlate poorly with the volume drained and changes in breathlessness. This suggests that loss of lung volume is an unlikely basis for breathlessness in patients with pleural effusion. However, there is only limited information on the effect of pleural effusions and pleural drainage on cardiorespiratory physiology [6-12], breathing mechanics (including diaphragmatic function) [13-16] and clinical outcomes. Our previous review confirmed that most published studies are small $(\mathrm{n}<35)$ and are focused on specific patient subgroups, and on particular aetiological factor(s) in isolation [17].

Drainage of pleural fluid is performed every day globally to relieve breathlessness. However, the severity of breathlessness at baseline often correlates poorly with the size of the effusion before drainage [18]; conversely, symptom reduction from fluid drainage varies [3-5]. The recent validation of a visual analogue scale (VAS) [19] to quantify breathlessness in patients with pleural effusion allows us to establish if a patient has a clinically meaningful improvement after fluid drainage and thus may guide patient selection.

The PLeural Effusion And Symptom Evaluation (PLEASE) study was the largest and most comprehensive to date and aimed to provide: 1) prospectively-captured data on the effect of pleural effusion (and its drainage) on cardiorespiratory, functional, radiographic and diaphragmatic parameters from an unbiased cross-sectional cohort of patients with a moderate to large effusion that required drainage; 2) data on the proportion of patients who have improvement in breathlessness from fluid drainage while exploring parameters that may predict response.

\section{Methods}

The study is a prospective single centre study where a centralised pleural service serves all (medical and surgical) specialties. The protocol has been published [20] and is summarised below. This study has been approved by the Sir Charles Gairdner and Osborne Park Group ethics committee (2014-079) and registered at the Australian New Zealand Clinical Trial Registry (ACTRN12616000820404). All participants gave signed informed consent.

\section{Participants}

All patients with a symptomatic pleural effusion requiring therapeutic pleural drainage as part of their clinical management, as decided by the treating physician, were identified as potential study participants. Patients requiring diagnostic (small volume) aspiration were not included. Exclusion criteria included age $<18$ years, pregnancy or lactation, requiring urgent pleural drainage before pre-drainage assessment could be completed, unable to perform two or more of the pre-drainage assessment tests, or unable to comply with the protocol. It was intended that all participants would have assessment of breathlessness and that spirometry, a 6-min walk test (6-MWT) and thoracic ultrasound would be administered.

\section{Study measurements}

Baseline demographics, smoking status, comorbidities (especially cardiopulmonary diseases) and Eastern Cooperative Oncology Group (ECOG) performance status were recorded. Chest radiography and baseline blood tests were performed as part of routine workup before pleural intervention. Pre-drainage measurements included patient-reported scores of breathlessness, vital signs (heart rate, respiratory rate and oxygen saturation), spirometry, 6-MWT and bedside ultrasonography to document the diaphragm

Affiliations: ${ }^{1}$ Dept of Respiratory Medicine, The Northern Hospital, Melbourne, Australia. ${ }^{2}$ Pleural Medicine Unit, Institute for Respiratory Health, Perth, Australia. ${ }^{3}$ Centre for Respiratory Health, School of Medicine and Pharmacology, University of Western Australia, Perth, Australia. ${ }^{4}$ Dept of Respiratory Medicine, Sunshine Coast University Hospital, Birtinya, Australia. ${ }^{5}$ Dept of Respiratory Medicine, Sir Charles Gairdner Hospital, Perth, Australia. ${ }^{6}$ Dept of Radiology, Sir Charles Gairdner Hospital, Perth, Australia. ${ }^{7}$ Dept of Cardiovascular Sciences, University of Leicester, Leicester, UK. ${ }^{8}$ School of Population and Global Health, University of Western Australia, Perth, Australia. ${ }^{9}$ West Australian Sleep Disorders Research Institute, Perth, Australia.

${ }^{10}$ Centre for Sleep Science, School of Human Sciences, University of Western Australia, Perth, Australia.

${ }^{11}$ Pulmonary Physiology and Sleep Medicine, Sir Charles Gairdner Hospital, Perth, Australia. ${ }^{12}$ Physiotherapy Unit, Institute for Respiratory Health, Perth, Australia. ${ }^{13}$ Physiotherapy Dept, Sir Charles Gairdner Hospital, Perth, Australia. ${ }^{14}$ School of Physiotherapy and Exercise Science, Curtin University, Perth, Australia. ${ }^{15}$ Joint first authors.

Correspondence: Y.C. Gary Lee, School of Medicine, UWA, 533 Harry Perkins Building, QE II Medical Centre, Perth, WA 6009, Australia. E-mail: gary.leeduwa.edu.au 
shape and movement. Three validated patient-reported breathlessness scores were employed: the VAS $[19,21]$, the dyspnoea-12 (D12) questionnaire [22-24] and the modified Borg 0-10 scale [25].

The VAS score is a validated measure of breathlessness in malignant pleural effusions [19] and has been used in studies of pleural drainage [26-29]. In this study, a standard script was used to explain to all participants how to mark the $100 \mathrm{~mm}$ vertical scale, as follows "To enable you to best describe how good or bad your shortness of breath is on a given day we have drawn a scale on which the best state you can imagine is marked 100 and the worst you can imagine is marked 0". The minimal clinically important difference (MCID) was set at $14 \mathrm{~mm}$, the lower limit of the $95 \%$ confidence interval (CI) of the MCID [19].

The D12 questionnaire assesses severity of breathlessness [24] with seven questions on physical components and five on affective components, each scored from zero to three. A maximum score of 36 points indicates the severest level of breathlessness. It was developed in people with chronic lung diseases and chronic heart failure [24], and validated in chronic obstructive pulmonary disease (COPD), asthma, lung fibrosis and pulmonary arterial hypertension (PAH) cohorts [22-24, 30].

The modified Borg 0-10 scale was used to assess breathlessness before the 6-MWT and the peak level of breathlessness during it [25]. If the patient required oxygen in the pre-drainage 6-MWT, supplementary oxygen at the same flow rate was provided for the post-drainage test. The 6-MWTs were performed by trained operators following international guidelines [31].

Spirometry was performed following the American Thoracic Society (ATS) recommendations [32].

Diaphragm shape and movement were assessed in an upright position while seated and was performed bilaterally (with the normal side being studied first) using bedside ultrasonography (Logiq-E, GE Healthcare, Chicago, IL, USA). A curvilinear probe $(2-5 \mathrm{MHz})$ was used to assess diaphragm shape and movement. The ultrasound probe was applied vertically or in an oblique transverse plane in the intercostal space, with acoustic coupling achieved using conventional gel between the transducer and the skin. B-mode ultrasound was performed by placing the probe at the posterior chest/posterior axillary line to assess diaphragmatic shape and movement. Shape was classified as normal (dome-shaped), flattened or everted. Diaphragm movement was considered normal if it moved downwards on inspiration (and vice versa), reduced when the amplitude of movement was lower than that of the contralateral hemi-diaphragm, or abnormal (either absent or paradoxical movements). Reduced diaphragm movement was assessed qualitatively by comparing it to the contralateral side. Measurements of each participant were conducted by the same operator both pre-procedure and $24-36 \mathrm{~h}$ post-procedure with the same ultrasound machine.

De-identified radiographs were assessed independently by a pulmonologist ( $\mathrm{R}$. Thomas) and a thoracic radiologist (Y.J. Kuok) in random order and in one large pool to avoid bias. The size of effusions on chest radiographs was graded as zero (no effusion), one (blunting of costophrenic angle), two ( $<25 \%$ but more than blunting), three ( $25 \%$ but $>50 \%$ of hemithorax), four $(50-75 \%$ of hemithorax) or five $(>75 \%$ of hemithorax) as per Light et al. [33]. Fluid loculations and/or mediastinal shift were recorded as "present" or "absent". Loculations were defined by any of the following: 1) fixed fluid in abnormal locations; 2) airfluid levels within the effusion; or 3) irregular scalloped appearance of the effusion contour [34]. The mean scores of the assessors were rounded to the nearest grade. In order to reach an agreed score by consensus, the two graders discussed any particular case if their initial scores differed by more than one grade for effusion size or if classification of loculation and/or mediastinal shift differed. Trapped lung in this study was defined as air or fluid in the pleural space occupying $\geqslant 25 \%$ of the lateral chest wall on chest radiograph post-procedure and/or if patient experienced chest pain and/or cough during pleural fluid drainage [28].

\section{Procedure}

The type of pleural drainage was determined by the attending clinician. In participants with bilateral pleural effusions, the side with the larger effusion size was drained and pre-drainage and post-drainage tests were performed prior to any drainage of the contralateral effusion. The macroscopic appearance of the fluid, the total volume drained and the fluid biochemistry (e.g. levels of protein, lactate dehydrogenase $(\mathrm{LDH})$ and $\mathrm{pH}$ ) were recorded.

\section{Post-drainage assessments}

Prior studies showed that gas exchange, lung compliance and respiratory resistance could worsen in the first few hours post-thoracentesis [6, 9, 35-37] and improvements were more consistent by $24 \mathrm{~h}[5,8,11,38]$. In this study, breathlessness scores, spirometry, 6-MWT and diaphragm ultrasound were repeated between 24 and $36 \mathrm{~h}$ post-drainage. This also diminished the impact of pain from the procedure on assessments. 


\section{Statistical methods}

Data were analysed using the R-environment for statistical computing (www.r-project.org) [39] using paired t-tests, estimated mean differences and 95\% CIs where appropriate. Logistic regression was used to determine which baseline factors were related to the response of a clinically important change in VAS scores. Potential explanatory variables were considered from demographics, as well as clinical, physiological and radiological variables, with univariate and multivariate odds ratios (ORs) and 95\% CIs. A separate set of analyses was carried out to examine which subsets of predictors of a clinically important change in VAS scores were important baseline predictors. Logistic regression was used and sensitivities, specificities, positive predictive values (PPVs) and negative predictive values (NPVs), along with C statistics, were calculated for the best model within each subset of explanatory variables and overall (see supplementary table E2). Model selection was carried out using backward Akaike's information criteria (AIC) and final models were determined by those models that minimised these criteria, such that only significant variables were left in the final models.

\section{Results}

Over 22 months from February 2015, 150 participants were enrolled (figure 1). Five were unable to perform post-drainage tests and their data were excluded from the final analyses. A small number of participants were unable to complete the spirometry (pre-drainage $(n=5)$, post-drainage $(n=10))$ or 6-MWT (pre-drainage $(n=19)$, post-drainage $(n=23))$ due to a variety of reasons.

\section{Demographics}

The median age of participants was 69 years (interquartile range (IQR) 30-92 years) and 55\% of participants were male. The most common underlying causes of the effusions were malignancy (63\%) and cardiac failure (15\%). In addition, $57 \%$ of patients had previously been treated by fluid drainage. The effusion was right-sided in $51 \%$, left-sided in $32 \%$ and bilateral in $17 \%$ of cases. Drainage was most commonly performed via an intercostal chest tube (44\%) and by needle aspiration (28\%) (table 1). Most of the participants $(n=135)$ had a moderate-to-large effusion (grade 3 or above) on chest radiograph and baseline mean VAS score was $43.7 \pm 21.0 \mathrm{~mm}$ before pleural fluid drainage (table 2).

\section{Differences between pre- and post-drainage parameters}

The median volume drained was $1.68 \mathrm{~L}$ (IQR 1.10-2.60 L). Drainage improved radiographic appearance. Most participants (93\%) had effusions of grades 3-5 pre-drainage whereas after drainage the majority (84\%) had grades of $0-2$. Median (IQR) grade improved from grade $3(3.0-4.0)$ at baseline to grade 2 (2.0-2.0) post-drainage (median difference $-1.0,95 \%$ CI -2.0 to $-1.0 ; \mathrm{p}<0.0001$ ).

\section{Breathlessness parameters}

Drainage improved scores on all of the breathlessness instruments used (see table 2 and figure 2). Mean VAS score for breathlessness improved from $43.7 \pm 21.0 \mathrm{~mm}$ at baseline to $71.6 \pm 17.7 \mathrm{~mm}$ post-drainage. The estimated mean difference of $28.0 \mathrm{~mm}$ (95\% CI 24.0-31.9), p<0.0001 was above the MCID cut-off of $14 \mathrm{~mm}$ [19].

The overall D12 score decreased from $19.2 \pm 9.7$ to $8.6 \pm 7.3$ post-drainage (estimated mean difference -10.5 , 95\% CI -12.0 to $-9.0 ; \mathrm{p}<0.0001)$. Improvements were observed in both components of D12: mean reduction was $6.4(95 \%$ CI 5.5-7.2) in the physical score and 4.2 (95\% CI 3.5-4.9) in the affective score (both $\mathrm{p}<0.0001$ ).

FIGURE 1 Flow chart describing screening and recruitment in the trial. Three participants deteriorated clinically prior to drainage being performed and two declined/were unable to perform any of the tests post-drainage.

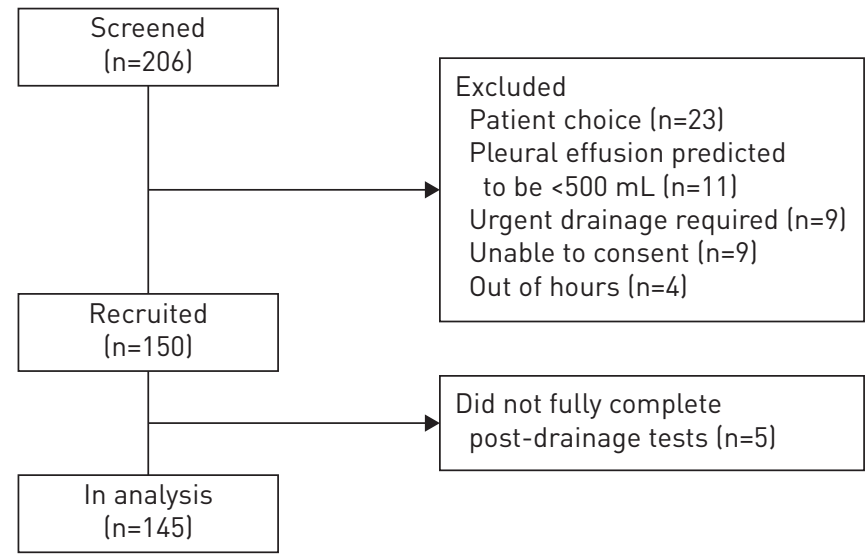


TABLE 1 Patient demographics

Characteristics

Subjects
Age years
Male
Comorbidities
Pulmonary
Cardiac
Renal
Hypoalbuminemia
Liver cirrhosis
Diabetes mellitus
Previous drainages
Aetiology of the pleural effusion
Malignant
$\quad$ Mesothelioma
NSCLC (lung)
Breast
Ovarian/endometrial
Lymphoma
Benign
Heart failure related
Side of pleural effusion
Right
Left
Bilateral
Procedure type
Intercostal catheter
Indwelling pleural catheter
Needle aspiration
Pleuroscopy
Volume of pleural fluid drained L
Trapped lung

Patients

145

$67.3 \pm 11.9$

$80(55)$

49 (34)

$77(44)$

$20(14)$

$17(12)$

$10(7)$

$28(19)$

83 (57)

92 (63)

37

17

9

9

5

53 (37)

20

$74(51)$

47 (32)

$24(17)$

$61(42)$

$41(28)$

$40(28)$

4 (3)

$1.67(1.10-2.60)$

$31(21)$

Data are presented as $\mathrm{n}$, mean $\pm \mathrm{SD}, \mathrm{n}(\%)$ or median (interquartile range). NSCLC: nonsmall cell lung cancer. \#: paramalignant $(n=6)$, carcinoma of unknown origin $(n=3)$, transitional cell carcinoma $(n=1)$, melanoma ( $n=1)$, small cell carcinoma $(n=1)$, oesophageal carcinoma $(n=1)$, colorectal carcinoma $(n=1)$, osteosarcoma $(n=1)$; ": pleural infection $(n=8)$, hepatic hydrothorax $(n=6)$, pleuritis $(n=4)$, aetiology not defined $(n=3)$, reactive effusion post-haemathorax $(n=2)$, yellow nail syndrome $(n=2)$, related to peritoneal dialysate $(n=1)$, pancreatitis ( $n=1)$, post-coronary artery bypass grafting effusion $(n=1)$, chylothorax $(n=2)$, tuberculous effusion $(n=1)$, post-lobectomy effusion $(n=1)$, benign asbestos-related pleural effusion $(n=1)$.

The mean modified Borg score at rest (before the 6-MWT) was $2.1 \pm 1.6$ and this improved to $1.4 \pm 1.2$, a mean decrease of 0.6 (95\% CI $0.3-0.9), \mathrm{p}<0.0001$. There was also a significant improvement in the mean modified Borg score after exertion (after the 6-MWT) from $4.5 \pm 2.6$ pre-drainage to $2.8 \pm 1.6$ post-drainage, a mean decrease of 1.6 (95\% CI 1.3-2.0), $\mathrm{p}<0.0001$. There was no significant correlation between change in distance walked and change in modified Borg score pre-6-MWT and post-6-MWT $(\mathrm{p}=0.7630)$.

There was a negative association between change in VAS and D12 score (meaning the same direction of change); however, no significant association was seen between change in VAS or D12 score with the Borg score pre-6-MWT and post-6-MWT (see supplementary table E1).

\section{Physiological parameters}

Heart and respiratory rates showed modest decreases post-drainage that were of statistical but uncertain clinical significance (table 2). The respiratory rate decreased from $20.4 \pm 2.9$ to $18.2 \pm 2.3$ breaths $\mathrm{min}^{-1}$ (mean difference 2.2, 95\% CI 1.7-2.8), $\mathrm{p}<0.0001$. The heart rate decreased from $89.8 \pm 17.3$ to $85.3 \pm 15.7$ beats. $\mathrm{min}^{-1}$ (mean difference 4.5, 95\% CI 2.3-6.6), $\mathrm{p}<0.0001$. Oxygen saturation did not change significantly and pre-drainage was $95.0 \pm 2.2 \%$ compared with a post-drainage value of $95.2 \pm 2.6 \%(\mathrm{p}=0.29)$.

Functional capacity

The 6-min walk distance (6-MWD) improved significantly (table 2 and figure 2) from $233 \pm 147 \mathrm{~m}$ pre-drainage to $263 \pm 161 \mathrm{~m}$ post-drainage. The mean increase of $29.7 \mathrm{~m}$ (95\% CI 17.6-41.8) exceeds the MCIDs used in many studies of lung diseases [40, 41]. 
TABLE 2 Breathlessness scores pre-drainage and post-drainage of pleural fluid

\begin{tabular}{|c|c|c|c|c|}
\hline & Pre-drainage & Post-drainage & Difference & p-value \\
\hline \multicolumn{5}{|l|}{ Breathlessness scores } \\
\hline VAS & $43.7 \pm 21.0$ & $71.6 \pm 17.7$ & $28.0(24.0-31.9)$ & $<0.0001$ \\
\hline D12 (overall) & $19.2 \pm 9.7$ & $8.6 \pm 7.3$ & $-10.5(-12.0$ to -9.1$)$ & $<0.0001$ \\
\hline D12 affective & $6.5 \pm 4.7$ & $2.3 \pm 3.3$ & $-4.2(-4.9$ to -3.5$)$ & $<0.0001$ \\
\hline D12 physical & $12.7 \pm 5.7$ & $6.3 \pm 4.6$ & $-6.4(-7.2$ to -5.5$)$ & $<0.0001$ \\
\hline Modified Borg (pre-6-MWT) & $2.1 \pm 1.6$ & $1.4 \pm 1.2$ & $-0.6(-0.9$ to -0.3$)$ & $<0.0001$ \\
\hline Modified Borg (post-6-MWT) & $4.5 \pm 2.6$ & $2.8 \pm 1.6$ & $-1.6(-2.0$ to -1.3$)$ & $<0.0001$ \\
\hline \multicolumn{5}{|l|}{ Functional capacity } \\
\hline 6-MWD m & $233 \pm 147$ & $263 \pm 161$ & $30(18-42)$ & $<0.0001$ \\
\hline \multicolumn{5}{|l|}{ Cardiorespiratory status } \\
\hline Heart rate beats $\cdot \mathrm{min}^{-1}$ & $89.8 \pm 17.3$ & $85.3 \pm 15.7$ & $-4.5(-6.6$ to -2.3$)$ & $<0.0001$ \\
\hline Respiratory rate breaths $\cdot \mathrm{min}^{-1}$ & $20.4 \pm 2.9$ & $18.2 \pm 2.3$ & $-2.2(-2.8$ to -1.7$)$ & $<0.0001$ \\
\hline $\mathrm{S}_{\mathrm{pO}_{2}} \%$ & $95.0 \pm 2.2$ & $95.2 \pm 2.6$ & $0.2(-0.2$ to 0.6$)$ & 0.29 \\
\hline \multicolumn{5}{|l|}{ Spirometry } \\
\hline $\mathrm{FEV}_{1} \%$ predicted & $43.9 \pm 15.3$ & $52.2 \pm 16.9$ & $8.2(6.6-9.9)$ & $<0.0001$ \\
\hline FVC $\%$ predicted & $45.4 \pm 17.0$ & $53.5 \pm 17.3$ & $8.0(6.2-9.9)$ & $<0.0001$ \\
\hline
\end{tabular}

Data are presented as mean \pm SD or mean difference $(95 \% \mathrm{CI})$, unless otherwise stated. The spirometry measures were based on the following participants: pre-drainage $(n=140)$, post-drainage $(n=135)$; the mean difference was based on 133 participants. The 6-min walk test (6-MWT) was based on the following participants: pre-drainage $(n=126)$, post-drainage $(n=122)$; the mean difference was based on 127 participants. The p-values were calculated using paired t-tests. VAS: visual analogue scale; D12: dyspnoea-12; 6-MWD: 6-min walk distance; $\mathrm{S}_{\mathrm{pO}_{2}}$ : oxygen saturation measured by pulse oximetry; $F \mathrm{VV}_{1}$ : forced expiratory volume in $1 \mathrm{~s}$; FVC: forced vital capacity.
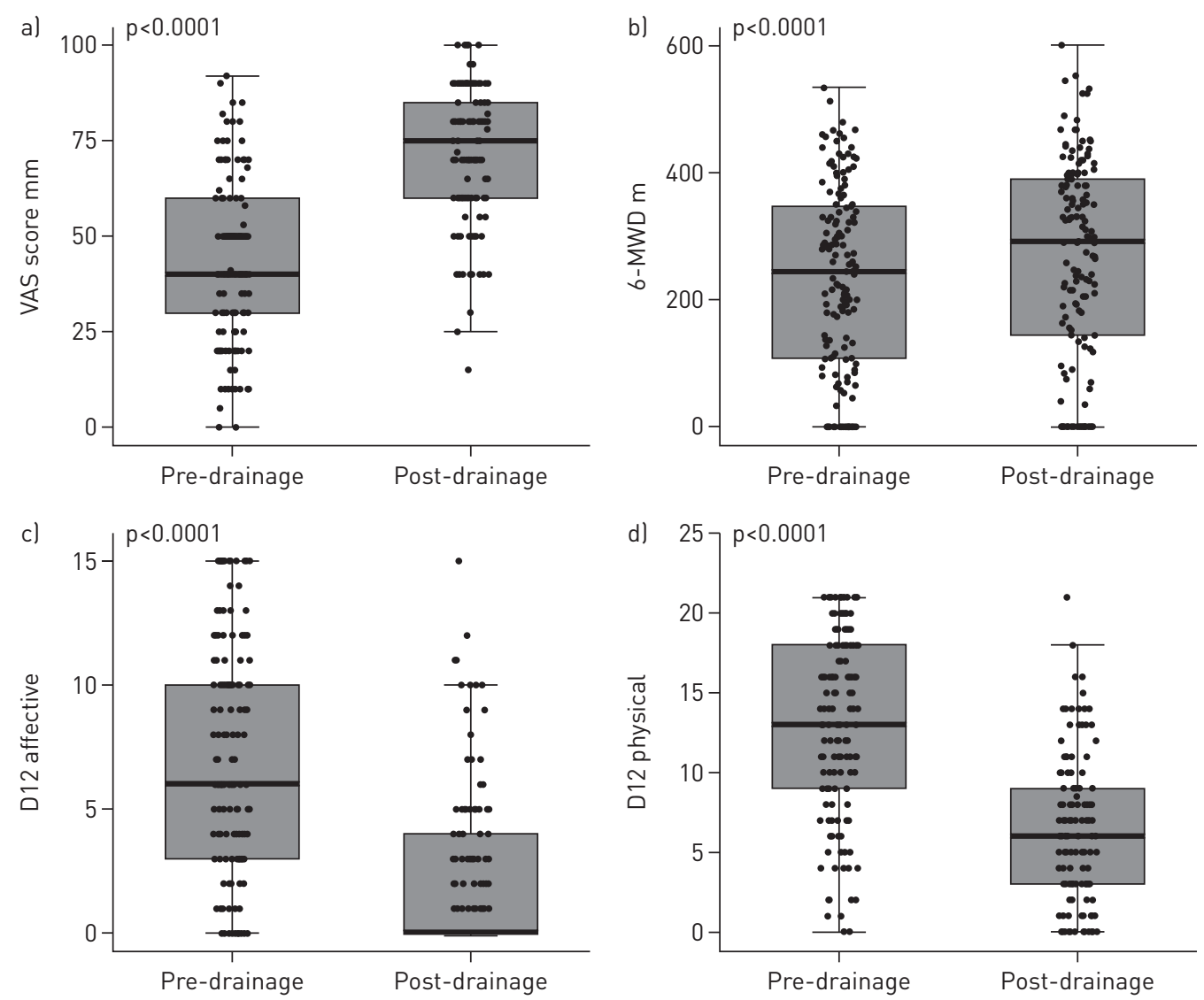

FIGURE 2 Comparisons of a) visual analogue scale (VAS) score; b) 6-min walk distance (6-MWD); c) dyspnoea-12 (D12) affective score; and d) D12 physical score, both pre-drainage and post-drainage. The $\mathrm{p}$-values provided are calculated using paired t-tests. 
TABLE 3 Change in diaphragmatic appearance and movement from pre-drainage to post-drainage using bedside ultrasonography

Appearance pre-drainage

Appearance post-drainage

\begin{tabular}{|c|c|c|c|c|}
\hline & Domed & Flattened & Everted & Total \\
\hline Domed & 73 & 0 & 0 & 73 \\
\hline Flattened & 45 & 4 & 0 & 49 \\
\hline Everted & 23 & 0 & 0 & 23 \\
\hline Total & 141 & 4 & 0 & 145 \\
\hline \multirow[t]{2}{*}{ Movement pre-drainage } & \multicolumn{4}{|c|}{ Movement post-drainage } \\
\hline & Normal & Reduced & $\begin{array}{c}\text { Abnormal/no movement/ } \\
\text { paradoxical }\end{array}$ & Total \\
\hline Normal & 40 & 5 & 0 & 45 \\
\hline Reduced & 18 & 12 & 0 & 30 \\
\hline Abnormal/no movement/paradoxical & 51 & 17 & 2 & 70 \\
\hline Total & 109 & 34 & 2 & 145 \\
\hline
\end{tabular}

\section{Lung function}

Improvement in spirometry was disproportionately small relative to the volume of fluid removed. Forced expiratory volume in $1 \mathrm{~s}\left(\mathrm{FEV}_{1}\right)$ increased by $0.22 \mathrm{~L}(8.2 \%(95 \% \mathrm{CI}$ 6.6-9.9) of individual's predicted values) from $1.19 \pm 0.48 \mathrm{~L}$ to $1.43 \pm 0.55 \mathrm{~L}(\mathrm{p}<0.001)$. Forced vital capacity (FVC) increased by $0.30 \mathrm{~L}(8.0 \%$ (95\% CI 6.2-9.9) of individual's predicted values) from $1.64 \pm 0.69 \mathrm{~L}$ to $1.95 \pm 0.76 \mathrm{~L}$ ( $\mathrm{p}<0.001$ ) (table 2 ).

\section{Diaphragm assessment}

Pre-drainage, $50 \%$ of participants had flattened or everted ultrasonographic appearances to their ipsilateral hemi-diaphragms (table 3). Post-drainage, most (94\%) had a normal dome-shaped appearance of the hemi-diaphragm. A significant proportion of participants had clinically meaningful improvement in breathlessness scores post-drainage (figure 3), regardless of whether their diaphragm appeared normal, everted or flattened in shape before drainage.

Movement of the ipsilateral hemi-diaphragm was normal in $31 \%$ of cases, reduced in $21 \%$ and abnormal in $48 \%$ pre-drainage. Almost all participants (97\%) with abnormal movement of the hemidiaphragm pre-drainage had an improvement to normal $(73 \%)$ or reduced $(24 \%)$ movement post-drainage. Thirty participants had reduced hemi-diaphragm movement at baseline and post-drainage 18 had normal movement and 12 had persistently reduced movement (table 3). A significant proportion of participants with reduced or abnormal diaphragm movement pre-drainage had clinically meaningful improvement in breathlessness scores post-drainage (figure 4).

There was a significant association between diaphragm movement and appearance pre-drainage $(\mathrm{p}<0.0001)$. The majority of participants with abnormal movement, no movement or paradoxical

FIGURE 3 Change in visual analogue scale (VAS) by diaphragmatic appearance pre-drainage. Percentages given describe the proportion of participants that had a change in VAS of $14 \mathrm{~mm}$ or more.

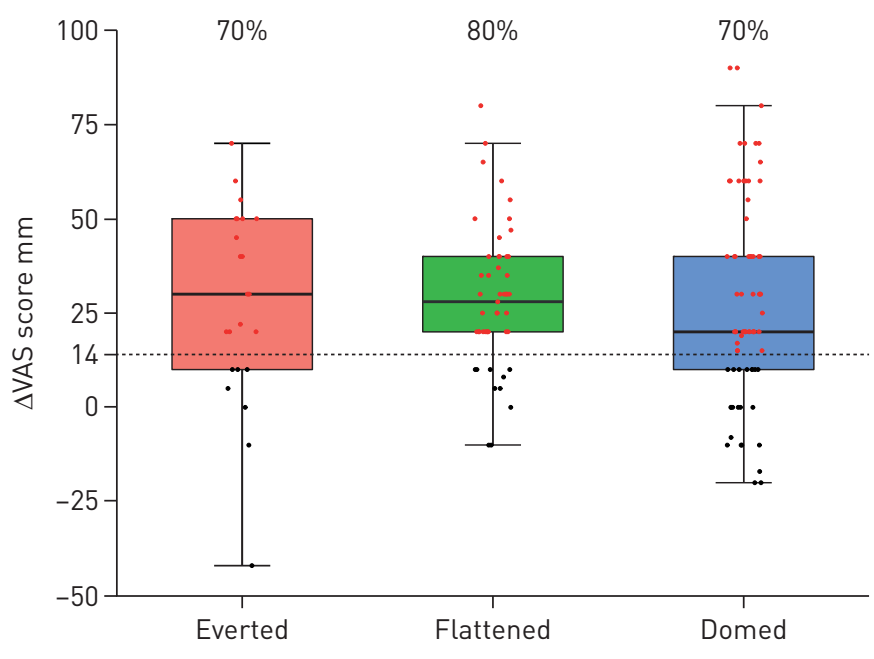


FIGURE 4 Change in visual analogue scale (VAS) by diaphragmatic movement pre-drainage. Percentages given describe the proportion of participants that had a change in VAS of $14 \mathrm{~mm}$ or more.

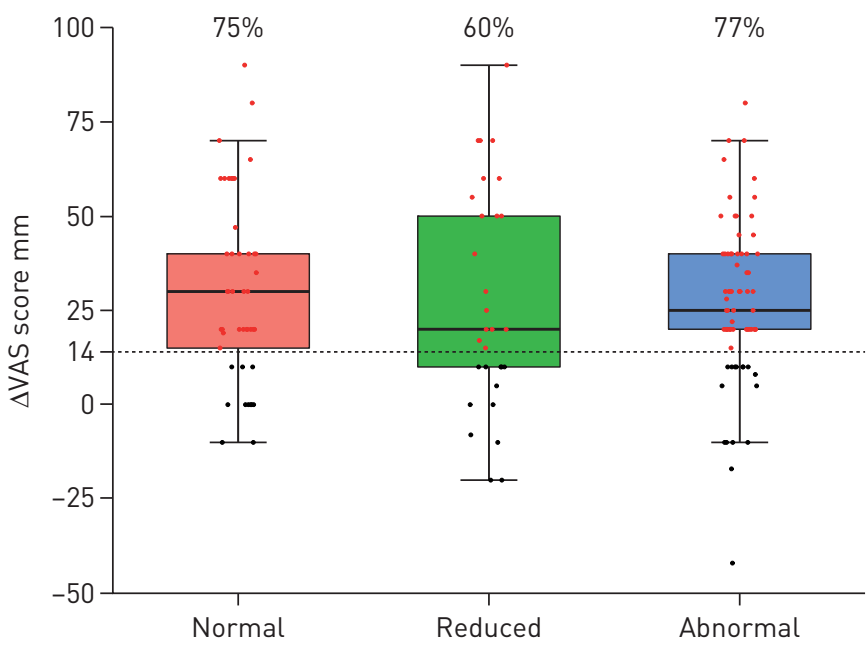

diaphragm movement had flattened (53\%) or everted (30\%) hemi-diaphragm shape pre-drainage. Most participants (84\%) with normal hemi-diaphragm movement had a domed-shaped hemi-diaphragm. The majority of participants (77\%) who returned to a domed-shaped hemi-diaphragm post-drainage had normal diaphragmatic movement (table 4).

\section{Symptom responders versus nonresponders}

After drainage, 106 participants (73\%) had clinically meaningful improvement in breathlessness $(\geqslant 14 \mathrm{~mm}$ increase in VAS score) (figure 5) and were termed the "responders". Of note, 26 out of 31 (84\%) of those with trapped lung responded versus $70 \%$ of those who did not have trapped lung (mean difference 0.14 , 95\% CI 10.02-0.29; $\mathrm{p}=0.13$ ).

In the univariate analysis (table 5), the responders were significantly more breathless at baseline compared with the nonresponders (OR 0.26, 95\% CI 0.15-0.44; $\mathrm{p}<0.0001$ ). In the multivariate analysis (table 5), more severe breathlessness (lower VAS score) at baseline (OR 5.83 for each decrease in standard deviation (SD), 95\% CI 3.05-11.16; $\mathrm{p}<0.0001$ ), abnormal (absent or paradoxical) hemi-diaphragm movement pre-drainage versus normal/reduced movement (OR 4.37, 95\% CI 1.41-13.56; $\mathrm{p}=0.0106$ ), a benign versus malignant pleural effusion (OR 3.39, 95\% CI 0.93-12.32; $\mathrm{p}=0.06$ ), higher pleural fluid $\mathrm{pH}$ (OR for one SD increase $1.92,95 \%$ CI 1.12-3.27; $\mathrm{p}=0.017$ ), or higher serum albumin level (OR for one SD increase 1.73, 95\% CI $1.01-2.95 ; \mathrm{p}=0.044)$ were independently associated with significant improvement in breathlessness post-drainage.

There was a trend towards having more responders in the higher effusion grade, which was not statistically significant in the univariate and multivariate analysis in this particular study $(70 \%, 71 \%$ and $83 \%$ of

\section{TABLE 4 Concordance between change in diaphragmatic movement and appearance from} pre-drainage to post-drainage using bedside ultrasonography

\begin{tabular}{lcccc} 
Movement pre-drainage & \multicolumn{3}{c}{ Appearance pre-drainage } \\
\cline { 2 - 5 } & Domed & Flattened & Everted & Total \\
\hline Normal & 38 & 5 & 2 & 45 \\
Reduced & 23 & 7 & 0 & 30 \\
Abnormal/no movement/paradoxical & 12 & 37 & 21 & 70 \\
Total & 73 & 49 & 23 & 145 \\
\hline Movement post-drainage & & Appearance post-drainage & \\
\cline { 2 - 5 } & Domed & Flattened & Everted & Total \\
\hline Normal & 108 & 1 & 0 & 109 \\
Reduced & 31 & 3 & 0 & 34 \\
Abnormal/no movement/paradoxical & 2 & 0 & 0 & 2 \\
Total & 141 & 4 & 0 & 145 \\
\hline
\end{tabular}


FIGURE 5 Change in visual analogue scale (VAS) from pre-drainage to post-drainage. Red dots indicate those participants who had an increase of at least $14 \mathrm{~mm}$.

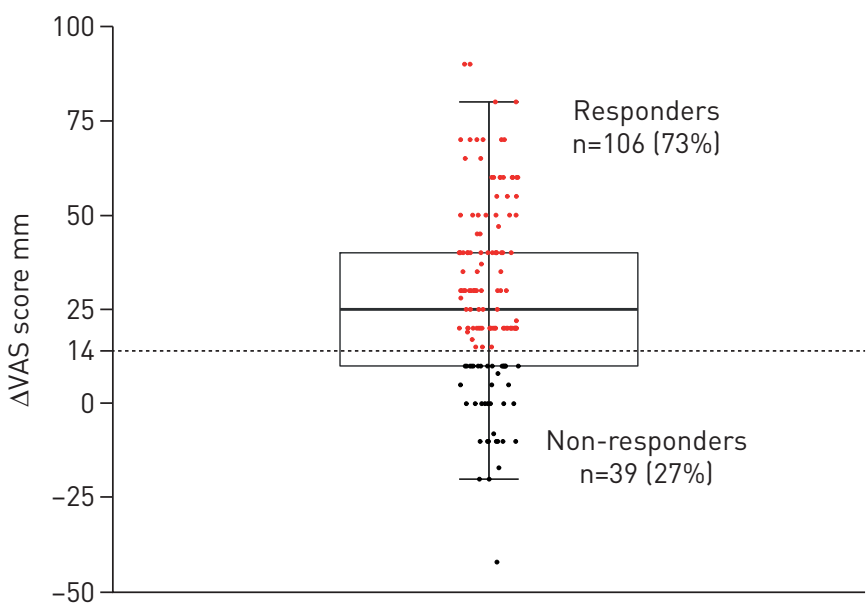

responders for effusion grades 3, 4 and 5 respectively). In terms of prediction of participants who would improve from pleural effusion drainage, all models were suboptimal (table 6 and figure 6). A model that included all variables provided the best predictive measures on the whole, with sensitivity $=0.82$, specificity $=0.78, \mathrm{PPV}=0.91, \mathrm{NPV}=0.61$ and area under the curve (AUC) $=0.85$ (table 6). The prediction value was largely driven by the baseline VAS score, which by itself would provide similar predictive power (sensitivity=0.87, specificity=0.61, PPV=0.86, NPV=0.63 and $\mathrm{AUC}=0.80$ ). Results using $9 \mathrm{~mm}$ and $19 \mathrm{~mm}$ cut-off values (see supplementary tables E3 and E4) or VAS as a continuous variable (see supplementary table E5) are also presented. Overall the main results and interpretation remain unaffected.

\section{Discussion}

The PLEASE study provides the largest and most comprehensive prospective dataset to date on symptoms, cardiorespiratory status, exercise capacity, radiographic and diaphragm features in an unselected cohort of patients with moderate-to-large pleural effusions necessitating fluid evacuation. Drainage of the effusion (median $1.68 \mathrm{~L}$ ) significantly improved breathlessness across all three instruments used (as well as exercise capacity) in the majority of participants. Reducing the space occupying or mass effect of pleural effusion appears to be an important contributor to improved breathlessness, although the mechanism is unclear. Lung volumes, heart rate and respiratory rate underwent only modest absolute changes after drainage, whereas the ipsilateral diaphragm underwent more obvious changes in shape and movement, possibly due to its more compressible/distortable nature. Abnormalities in diaphragm movement were common and their presence, in addition to baseline level of breathlessness, were independent predictors of likelihood of symptom relief after fluid drainage. These data help define the benefits clinicians/patients can expect from pleural fluid drainage and provide hypothesis-generating information on potential mechanisms of breathlessness in patients with pleural effusions.

Our findings help to clarify several misconceptions about the impact of pleural effusion. First, despite the moderate-to-large effusions (grade 3 and above) and severity of breathlessness, pre-drainage vital signs remained relatively unaffected, especially oxygen saturation (mean 95\%), suggesting that hypoxaemia is not the main driver of patients' breathlessness. These parameters showed only modest improvement post-drainage despite significant improvements in symptoms and 6-MWD. Clinicians should consider searching for concurrent/alternative causes of hypoxaemia (if such exist) even in patients with a sizeable effusion. Patients with breathlessness and a pleural effusion can have significant benefit (and should not be denied drainage) even if their vital signs and oxygenation are normal. These observations support suggestions that effusion-related breathlessness arises from alteration of respiratory mechanics and increased effort of breathing $[15,16]$.

Secondly, we found that traditional teaching is over-simplistic in attributing the benefits of pleural drainage to removal of extrinsic fluid compression (to allow lung expansion). Increases in spirometric lung volumes were small and correlated poorly with volume of fluid drained and the improvement in breathlessness. However, given the expected noise of measurement of lung volumes and VAS, it may be difficult to demonstrate a significant correlation between these measurements even if there was a mechanistic relationship. Nonetheless, our finding likely implies that lung compression was not the principle mechanism by which the often litres of pleural fluid were accommodated within the thoracic cage. It is likely that the thoracic cavity expands (akin to hyperinflation in COPD) to accommodate the additional volume. This is further supported by the observation that breathlessness improved in a similar 
TABLE 5 Measure of responders (those participants with an increase in visual analogue scale (VAS) of $\geqslant 14 \mathrm{~mm}$ ) by each explanatory variable, together with unadjusted and multivariable odds ratios (ORs) from logistic regression with response change in VAS ( $\mathrm{n}=145$ )

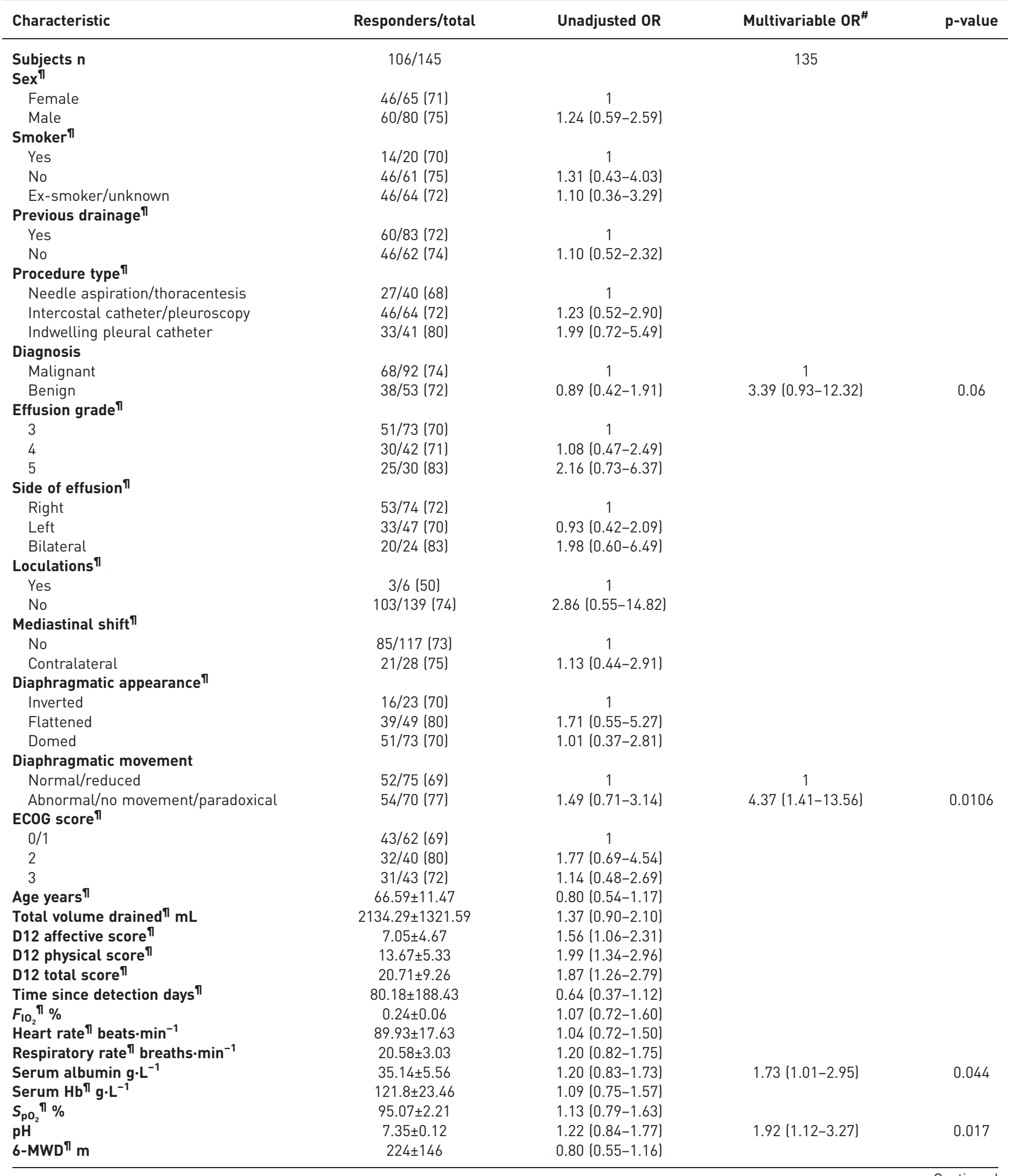


TABLE 5 Continued

\begin{tabular}{|c|c|c|c|c|}
\hline Characteristic & Responders/total & Unadjusted OR & Multivariable $\mathrm{OR}^{\#}$ & p-value \\
\hline FEV & $42.65 \pm 14.40$ & 0.75 (0.51-1.08) & & \\
\hline FVC $^{\Uparrow 1} \%$ predicted & $44.48 \pm 16.40$ & $0.82(0.57-1.19)$ & & \\
\hline
\end{tabular}

Data are presented as $\mathrm{n} / \mathrm{n}(\%)$, mean $\pm \mathrm{SD}$, or OR $(95 \% \mathrm{CI})$, unless otherwise stated. In the univariate analysis for logistic regression analyses comparing the responders and nonresponders, there was no difference in age, sex, diagnosis (whether malignant or benign), procedure type, previous drainage, radiographic and diaphragmatic appearance, ECOG performance status, volume drained, 6-min walk distance (6-MWD), serum albumin, or physiological and spirometric variables. The responders were significantly more breathless at baseline compared with the nonresponders (OR $0.26,95 \% \mathrm{Cl} 0.15-0.44 ; \mathrm{p}<0.0001$ ). In the multivariate analysis, baseline patient-reported breathlessness, diaphragm movement on ultrasonography, diagnosis (malignant or benign) and $\mathrm{pH}$ of the pleural fluid, as well as blood albumin level, were all independently associated with a significant improvement in breathlessness (increase in VAS score $\geqslant 14 \mathrm{~mm}$ ). Participants who reported more severe breathlessness at baseline (lower VAS scores) were more likely to have a positive response (OR 5.83 for each decrease in SD, $95 \% \mathrm{Cl}$ $3.05-11.16 ; p<0.0001$ ) as were those with abnormal labsent or paradoxical) hemi-diaphragm movement pre-drainage (OR $4.37,95 \% \mathrm{Cl} 1.41-$ 13.56; $\mathrm{p}=0.0106$ ) versus normal and reduced diaphragm movement and those who had a benign versus a malignant pleural effusion (OR 3.39 , $95 \% \mathrm{Cl}$ 0.93-12.32; $\mathrm{p}=0.06$ ). Participants were also more likely to have an improvement in breathlessness if they had higher pleural fluid $\mathrm{pH}$ at baseline (OR for one SD increase 1.92, 95\% Cl 1.12-3.27; $\mathrm{p}=0.017$ ) or higher serum albumin levels at baseline (OR for one sD increase $1.73,95 \%$ CI 1.01-2.95; $p=0.044$ ). All variables are baseline measures with the exception of volume drained. ORs for continuous variables are expressed as ORs for a 1 SD increase in the variable. ECOG: Eastern Cooperative Oncology Group; D12: dyspnoea-12; $F_{10}$ : inspiratory oxygen fraction; Hb: haemoblobin; $\mathrm{S}_{\mathrm{pO}_{2}}$ : oxygen saturation measured by pulse oximetry; $\mathrm{FEV}_{1}$ : forced expiratory volume in $1 \mathrm{~s}$; FVC: forced vital capacity. ${ }^{\#}$ : model selected by Akaike's information criteria ( $A I C)$ considering all variables modelling event as response (change in VAS $\geqslant 14$ mm); ${ }^{\text {: }}$ not in the final selected model.

proportion of patients with and without fully expandable lungs. Clinicians should not exclude patients from therapeutic thoracentesis on the basis of "trapped" (non-expandable) lung.

Thirdly, our study established that downward displacement of the dome of the hemi-diaphragm was common (resulting in its flattening/eversion), presumably to help accommodate the fluid. Reduced or abnormal (even paradoxical) diaphragm movement was common and such changes could render the diaphragm ineffective as a respiratory muscle. Drainage of the effusion improved diaphragm shape and movement. Indeed, patients with abnormal diaphragm movement were more likely to achieve symptom relief from drainage. In future, more sophisticated ways of assessing diaphragm function may help predict response to pleural drainage.

Breathlessness on exertion, rather than at rest, is often the first complaint in patients with a pleural effusion. In our study, the magnitude of functional impairment prior to drainage was similar to very severe stable COPD patients and the 6-MWDs were similar to COPD patients hospitalised for acute exacebration [42]. The mean improvement post-drainage was $30 \mathrm{~m}$ in our cohort, which matches the commonly applied MCID $(25 \mathrm{~m})$ in a COPD study [41]. Fluid drainage not only improved the 6-MWD but also the post-drainage Borg scores both before and after the 6-MWT. Interestingly, improvements in 6-MWD did not correlate with symptom improvement (VAS score), suggesting that other individual factors (e.g. concurrent comorbidity) may have a role in determining benefits.

A sizeable percentage of patients (27\%) did not experience meaningful improvement in breathlessness, a finding similarly to that of PsalLidas et al. [29]. In our study, individuals who were very breathless and/or had abnormal diaphragm movement at baseline were more likely to improve following drainage. However, the predictive models developed were not sufficiently accurate to be applied clinically. This highlights that breathlessness is a complex multifactorial process not easily predicted despite the comprehensive list of radiographic, physiologic and diaphragmatic variables we captured. Nonetheless, our data indicated that self-reported severity of breathlessness is a simple and useful guide for clinicians to decide on the need for fluid removal, presumably because it encompasses other complex variables that we could not/did not account for.

This study has limitations. First, it was conducted at a single centre with a centralised pleural service that captures investigations of patients under all subspecialties and minimises selection bias. This is supported by the fact that malignant and cardiac effusions formed the majority of the cohort, consistent with the commonly cited distribution of pleural effusions by aetiology. Secondly, $57 \%$ of participants had prior pleural fluid drainages and represented the "real-world" case mix. Amongst these, 23 out of 82 (28\%) did not have a clinically meaningful improvement in breathlessness $(<14 \mathrm{~mm}$ decrease in VAS score). The response rate was therefore similar in those with prior drainages and in the overall response rates. It is therefore less likely that individuals benefiting from prior drainages inflated the response rate. Thirdly, the 
TABLE 6 Comparison of various subsets of baseline characteristics to determine which provide the best model for prediction of change in visual analogue scale (VAS)

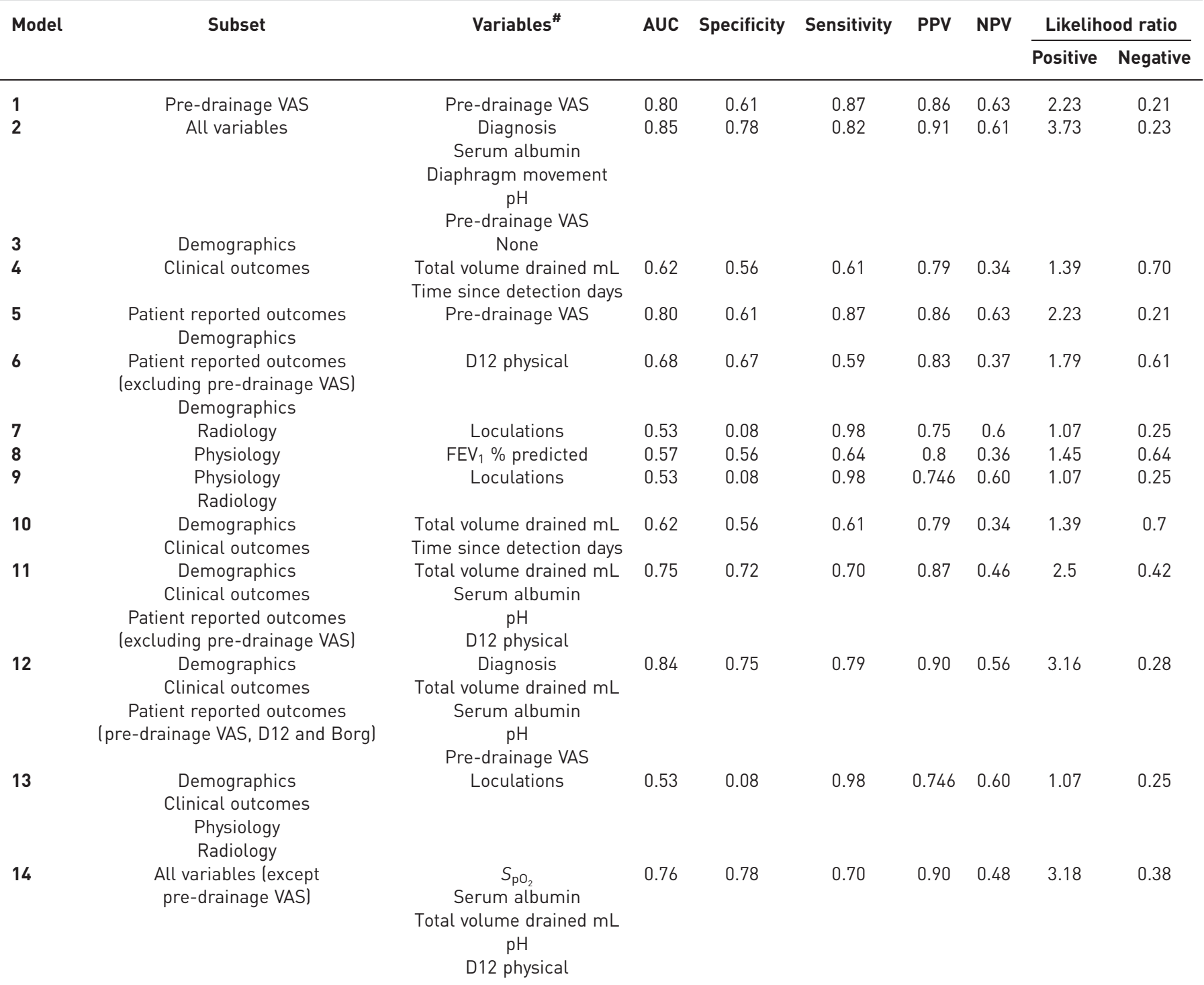

Models were fitted using logistic regression and a change of $14 \mathrm{~mm}$ or more as a positive response. A model that included all variables provided the best predictive measures on the whole, with sensitivity $=0.82$, specificity $=0.78, P P V=0.91, N P V=0.61$ and $A U C=0.85$. However, all models were suboptimal and many other models provided results close to this. The prediction value was largely driven by the baseline VAS score, which by itself would provide similar predictive power (sensitivity=0.87, specificity=0.61, PPV=0.86, NPV=0.63 and AUC=0.80). All variables were pre-drainage with the exception of total volume drained. A value of $n=135$ for complete cases for all variables was used in order to compare between models. All variables considered in each of the 14 models are described in table E2. AUC: area under the curve; PPV: positive predictive value; NPV: negative predictive value; D12: dyspnoea-12; $\mathrm{FEV}_{1}$ : forced expiratory volume in $1 \mathrm{~s} ; \mathrm{S}_{\mathrm{pO}_{2}}$ : oxygen saturation measured by pulse oximetry. " : as selected by Akaike's information criteria (AIC).

extensive nature of the post-test assessments precluded us from repeating the full investigations over a time course to establish the time point of maximal benefit (currently unknown). Prior literature suggested that there could be clinical deterioration in some physiological parameters in the first few hours post-drainage and therefore performing the post-drainage tests at $24-36 \mathrm{~h}$, though arbitrary, appeared sensible and bypassed confounders such as pain/discomfort from the initial procedures [29]. We demonstrated that at this time frame the majority of patients had good symptom reduction, a finding consistent across the three breathlessness instruments used. Fourthly, diaphragm assessment using ultrasound was performed qualitatively and more invasive measures of diaphragmatic function (e.g. trans-diaphragmatic pressure and diaphragm electromyography) may have provided useful information but were impractical in view of the number of other assessments in this study. Fifthly, depending on the clinical indications, not all patients 

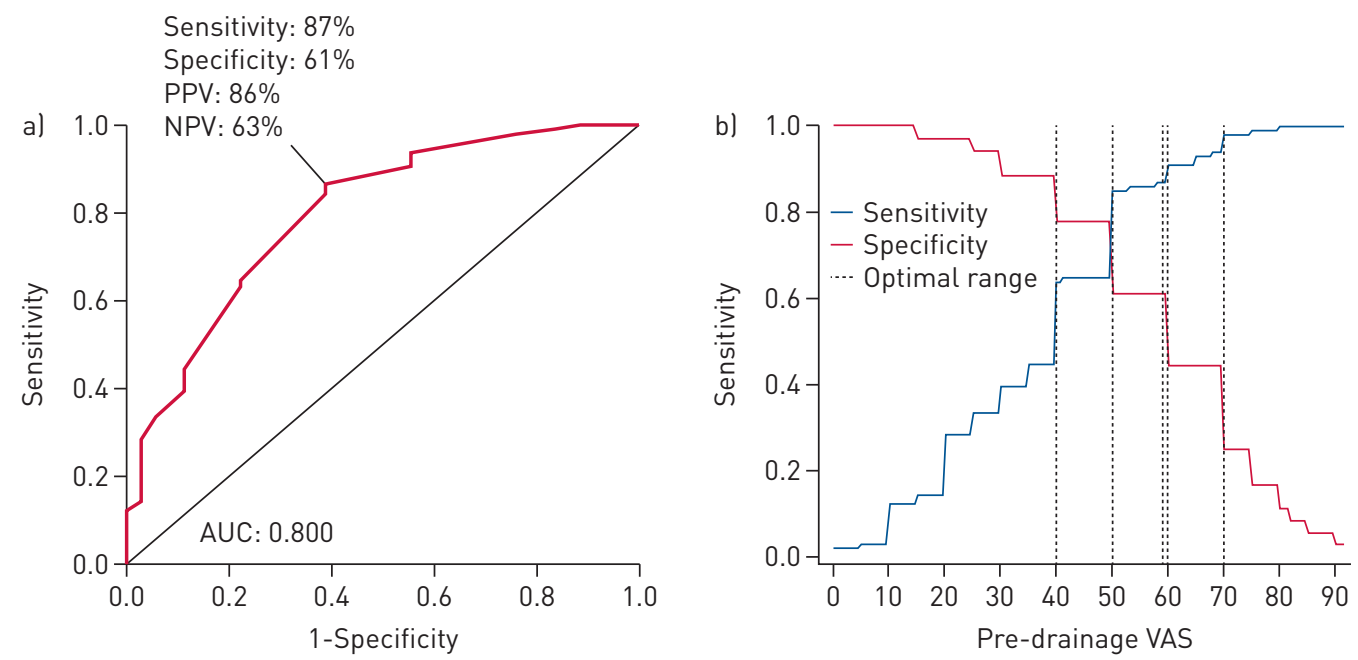

FIGURE 6 a) Receiver operating characteristic (ROC) curve for model 1 (pre-drainage visual analogue scale (VAS) onlyl as the "best" predictive model. b) Demonstration of how pre-drainage VAS score changes sensitivity and specificity. Lower values of pre-drainage VAS lead to higher specificity, while higher values of pre-drainage VAS lead to higher sensitivity. AUC: area under the curve; PPV: positive predictive value; NPV: negative predictive value.

had complete drainage of their effusion. Nevertheless, a significant volume (median $1.68 \mathrm{~L}$ ) was removed and radiographically most of the patients $(84 \%)$ had a post-drainage chest radiograph effusion grade of 0-2 only. Sixthly, the VAS is less repeatable when compared to the Borg score and may have a ceiling effect that likely explains why greater breathlessness pre-drainage is associated with greater reduction in breathlessness post-procedure. Reassuringly, two other instruments were used in our study and the modified Borg and D12 scores were congruent with VAS in their directions of change post-drainage. Seventhly, the determination of meaningful relief of breathlessness was based on an arbitrary level of change in VAS. The purpose of the MCID established by Mishra et al. [19] was to allow interpretation of whether a statistically significant effect on change in breathlessness was clinically meaningful between subjects in two randomised arms in a larger randomised controlled trial. It is uncertain if such an MCID threshold can be used to define each participant having a clinically meaningful change in breathlessness in order to justify a drainage procedure.

This study provides a platform for further investigations. In conclusion, drainage of pleural effusion improves symptoms, functional capacity and spirometry. In addition it normalises diaphragm shape and movement in the majority of participants. An underlying "trapped" (non-expandable) lung did not preclude symptom benefits from drainage. Patient-reported level of breathlessness is the best predictor of symptom benefit. Abnormal diaphragmatic function may be an important contributor to breathlessness in patients with pleural effusion and should be a topic for future research.

Acknowledgements: S. Muruganandan, M. Azzopardi and R. Thomas have received clinical research fellowships from the Western Australia Cancer and Palliative Care Network. R. Thomas is a National Health and Medical Research Council (NHMRC) and Cancer Council of Western Australia Early Career Fellow. Y.C.G. Lee held a National Health and Medical Research Council (NHMRC) Career Development Fellowship followed by a MRFF Practitioner Fellowship. He has received research project grant funding from the NHMRC, New South Wales Dust Disease Authority, Sir Charles Gairdner Research Advisory Committee and the Cancer Council of Western Australia. P.R. Eastwood is a National Health and Medical Research Council (NHMRC) Senior Research Fellow (number 1042341) and receives research project grant funding from the NHMRC, Sir Charles Gairdner Research Advisory Committee, Australia and New Zealand College of Anaesthetists, Princess Margaret Hospital Foundation and the WA Department of Health. S. Jenkins receives research project grant funding from the NHMRC and Sir Charles Gairdner Research Advisory Committee.

Author contributions: S. Muruganandan and M. Azzopardi are joint first authors. Y.C.G. Lee and R. Thomas conceived the initial trial concept with advice from B. Singh and P.R. Eastwood (diaphragm and respiratory physiology) and S. Jenkins (breathlessness scores and 6MWT). Together, these authors developed and modified the trial design and protocol. C.A. Read is the trial manager and oversees data collection and administrative matters. S. Muruganandan, R. Thomas, D.B Fitzgerald and M. Azzopardi were the trial coordinators, recruited patients and collected data. Y.J. Kuok and R. Thomas assessed the radiographs independently. H.M. Cheah assisted with database and data management. K. Murray and C.A. Budgeon were responsible for the statistical analyses. Y.C.G. Lee is the chief investigator and takes overall responsibility for all aspects of trial design, the protocol and trial conduct. All authors have read and approved the final manuscript. 
Support statement: This project has received project grant funding from the Cancer Council of Western Australia and the Sir Charles Gairdner Research Advisory Group. Funding information for this article has been deposited with the Crossref Funder Registry.

Conflict of interest: S. Muruganandan has nothing to disclose. M. Azzopardi has nothing to disclose. R. Thomas has nothing to disclose. D.B Fitzgerald has nothing to disclose. Y.J. Kuok has nothing to disclose. H.M. Cheah has nothing to disclose. C.A. Read has nothing to disclose. C.A. Budgeon has nothing to disclose. P.R. Eastwood has nothing to disclose. S. Jenkins has nothing to disclose. B. Singh has nothing to disclose. K. Murray has nothing to disclose. Y.C.G. Lee has served on the advisory board of CareFusion/BD Ltd and has previously led clinical trials for which Rocket Medical plc (UK) provided the drainage kits for study participants without charge, as well as providing an unrestricted educational grant to assist the running of the trial.

\section{References}

1 Marel M, Zrutova M, Stasny B, et al. The incidence of pleural effusion in a well-defined region: epidemiologic study in Central Bohemia. Chest 1993; 104: 1486-1489.

2 Roberts ME, Neville E, Berrisford RG, et al. Management of a malignant pleural effusion: British Thoracic Society pleural disease guideline 2010. Thorax 2010; 65: Suppl. 2, ii32-ii40.

3 Cartaxo AM, Vargas FS, Salge JM, et al. Improvements in the 6-min walk test and spirometry following thoracentesis for symptomatic pleural effusions. Chest 2011; 139: 1424-1429.

4 Estenne M, Yernault JC, De Troyer A. Mechanism of relief of dyspnea after thoracocentesis in patients with large pleural effusions. Am J Med 1983; 74: 813-819.

5 Wang JS, Tseng $\mathrm{CH}$. Changes in pulmonary mechanics and gas exchange after thoracentesis on patients with inversion of a hemidiaphragm secondary to large pleural effusion. Chest 1995; 107: 1610-1614.

6 Agusti AGN, Cardus J, Roca J, et al. Ventilation-perfusion mismatch in patients with pleural effusion: effects of thoracentesis. Am J Respir Crit Care Med 1997; 156: 1205-1209.

7 Goligher EC, Leis JA, Fowler RA, et al. Utility and safety of draining pleural effusions in mechanically ventilated patients: a systematic review and meta-analysis. Crit Care 2011; 15: R46.

8 Perpina M, Benlloch E, Marco V, et al. Effect of thoracentesis on pulmonary gas exchange. Thorax 1983; 38: 747-750.

9 Brown NE, Zamel N, Aberman A. Changes in pulmonary mechanics and gas exchange following thoracocentesis. Chest 1978; 74: 540-542.

10 Doelken P, Abreu R, Sahn SA, et al. Effect of thoracentesis on respiratory mechanics and gas exchange in the patient receiving mechanical ventilation. Chest 2006; 130: 1354-1361.

11 Razazi K, Thille AW, Carteaux G, et al. Effects of pleural effusion drainage on oxygenation, respiratory mechanics, and hemodynamics in mechanically ventilated patients. Ann Am Thorac Soc 2014; 11: 1018-1024.

12 Altschule MD, Zamcheck N. The effects of pleural effusion on respiration and circulation in man. J Clin Invest 1944; 23: 325-331.

13 Wang LM, Cherng JM, Wang JS. Improved lung function after thoracocentesis in patients with paradoxical movement of a hemidiaphragm secondary to a large pleural effusion. Respirology 2007; 12: 719-723.

14 Light RW, Stansbury DW, Brown SE. Changes in pulmonary function following therapeutic thoracocentesis (abstract). Chest 1981; 80: 341.

15 Cooper JC, Elliott ST. Pleural effusions, diaphragm inversion, and paradox: new observations using sonography. AJR Am J Roentgenol 1995; 164: 510.

16 Mulvey RB. The effect of pleural fluid on the diaphragm. Radiology 1965; 84: 1080-1086.

17 Thomas R, Jenkins S, Eastwood PR, et al. Physiology of breathlessness associated with pleural effusions. Curr Opin Pulm Med 2015; 21: 338-345.

18 Garske LA, Kunarajah K, Zimmerman PV, et al. In patients with unilateral pleural effusion, restricted lung inflation is the principal predictor of increased dyspnoea. PLoS One 2018; 13: e0202621.

19 Mishra EK, Corcoran JP, Hallifax RJ, et al. Defining the minimal important difference for the visual analogue scale assessing dyspnea in patients with malignant pleural effusions. PLoS One 2015; 10: e0123798.

20 Thomas R, Azzopardi M, Muruganandan S, et al. Protocol of the Pleural Effusion And Symptom Evaluation (PLEASE) study on the pathophysiology of breathlessness in patients with symptomatic pleural effusions. BMJ Open 2016; 6: e013213.

21 Ries AL. Minimally clinically important difference for the UCSD Shortness of Breath Questionnaire, Borg Scale, and Visual Analog Scale. COPD 2005; 2: 105-110.

22 Yorke J, Russell AM, Swigris J, et al. Assessment of dyspnea in asthma: validation of the Dyspnea-12. J Asthma 2011; 48: 602-608

23 Yorke J, Swigris J, Russell AM, et al. Dyspnea-12 is a valid and reliable measure of breathlessness in patients with interstitial lung disease. Chest 2011; 139: 159-164.

24 Yorke J, Moosavi SH, Shuldham C, et al. Quantification of dyspnoea using descriptors: development and initial testing of the Dyspnoea-12. Thorax 2010; 65: 21-26.

25 Borg G. Psychophysical bases of perceived exertion. Med Sci Sports Exerc 1982; 14: 377-381.

26 Davies HE, Mishra EK, Kahan BC, et al. Effect of an indwelling pleural catheter $v s$ chest tube and talc pleurodesis for relieving dyspnea in patients with malignant pleural effusion: the TIME2 randomized controlled trial. JAMA 2012; 307: 2383-2389.

27 Thomas R, Fysh ETH, Smith NA, et al. Effect of an indwelling pleural catheter $v s$ talc pleurodesis on hospitalization days in patients with malignant pleural effusion: the AMPLE randomized clinical trial. JAMA 2017; 318: 1903-1912.

28 Muruganandan S, Azzopardi M, Fitzgerald DB, et al. Aggressive versus symptom-guided drainage of malignant pleural effusion via indwelling pleural catheters (AMPLE-2): an open-label randomised trial. Lancet Respir Med 2018; 6: 671-680.

29 Psallidas I, Yousuf A, Talwar A, et al. Assessment of patient-reported outcome measures in pleural interventions. BMJ Open Respir Res 2017; 4: e000171. 
30 Yorke J, Armstrong I. The assessment of breathlessness in pulmonary arterial hypertension: reliability and validity of the Dyspnoea-12. Eur J Cardiovasc Nurs 2014; 13: 506-514.

31 Holland A, Spruit M, Troosters T, et al. An official European Respiratory Society/American Thoracic Society technical standard: field walking tests in chronic respiratory disease. Eur Respir J 2014; 44: 1428-1446.

32 Miller MR, Crapo R, Hankinson J, et al. General considerations for lung function testing. Eur Respir J 2005; 26: 153-161.

33 Light RW, Rogers JT, Cheng D, et al. Large pleural effusions occurring after coronary artery bypass grafting. Ann Intern Med 1999; 130: 891-896.

34 Himelman RB, Callen PW. The prognostic value of loculations in parapneumonic pleural effusions. Chest 1986; 90: 852-856.

35 Brandstetter RD, Cohen RP. Hypoxemia after thoracentesis. JAMA 1979; 242: 1060-1061.

36 Karetzky MS, Kothari GA, Fourre JA, et al. Effect of thoracentesis on arterial oxygen tension. Respiration 1978; 36: 96-103.

37 Brandstetter RD, Cohen RP. Hypoxemia after thoracentesis. A predictable and treatable condition. JAMA 1979; 242: $1060-1061$.

38 Talmor M, Hydo L, Gershenwald JG, et al. Beneficial effects of chest tube drainage of pleural effusion in acute respiratory failure refractory to positive end-expiratory pressure ventilation. Surgery 1998; 123: 137-143.

39 R: a language and environment for statistical computing. Vienna, R Foundation for Statistical Computing, 2017. www.r-project.org/ Date last accessed: 2 April 2020.

40 Bohannon RW, Crouch R. Minimal clinically important difference for change in 6-minute walk test distance of adults with pathology: a systematic review. J Eval Clin Pract 2017; 23: 377-381.

41 Holland AE, Hill CJ, Rasekaba T, et al. Updating the minimal important difference for six-minute walk distance in patients with chronic obstructive pulmonary disease. Arch Phys Med Rehabil 2010; 91: 221-225.

42 Tsai LLY, Alison JA, McKenzie DK, et al. Physical activity levels improve following discharge in people admitted to hospital with an acute exacerbation of chronic obstructive pulmonary disease. Chron Respir Dis 2016; 13: 23-32. 